ANNALES

POLONICI MATHEMATICI

$96.3(2009)$

\title{
Uniqueness of entire functions and their derivatives
}

\author{
by INDRAJIT LAHIRI (West Bengal) \\ and Gautam Kumar Ghosh (Bireswarpur)
}

\begin{abstract}
We study the uniqueness of entire functions which share a value or a function with their first and second derivatives.

1. Introduction, definitions and results. Let $f$ be a non-constant meromorphic function in the open complex plane $\mathbb{C}$. A meromorphic function $a=a(z)$ is called a small function of $f$ if $T(r, a)=S(r, f)$, where $T(r, f)$ is the Nevanlinna characteristic function of $f$ and $S(r, f)=o\{T(r, f)\}$ as $r \rightarrow \infty$ possibly outside a set of finite linear measure. Also we denote by $E(a ; f)$ the set of distinct zeros of $f-a$.

The problem of uniqueness of meromorphic functions sharing values with their derivatives is a special case of the uniqueness theory of meromorphic functions. This problem was initiated by Rubel and Yang [4] with the following result.
\end{abstract}

TheOrem A ([4]). Let $f$ be a non-constant entire function. If $f$ and $f^{\prime}$ share the values $a$ and $b$ counting multiplicities then $f \equiv f^{\prime}$.

Considering $f=e^{e^{z}} \int_{0}^{z} e^{-e^{t}}\left(1-e^{t}\right) d t$ we see that $f^{\prime}-1=e^{z}(f-1)$ and so the condition that $f$ and $f^{\prime}$ share two values is essential for Theorem A. In 1986 Jank, Mues and Volkman [3] considered the problem of sharing a single value by the derivatives of an entire function and proved the following result.

THEOREM B $([3])$. Let $f$ be a non-constant entire function and a $(\neq 0)$ be a finite number. If $E(a ; f)=E\left(a ; f^{\prime}\right)$ and $E(a ; f) \subset E\left(a ; f^{\prime \prime}\right)$ then $f \equiv f^{\prime}$.

In 2002 Chang and Fang [1] extended Theorem B and proved the following result.

2000 Mathematics Subject Classification: Primary 30D35.

Key words and phrases: entire function, derivative, uniqueness. 
TheOREM C ([1]). Let $f$ be a non-constant entire function. If $E(z ; f)=$ $E\left(z ; f^{\prime}\right)$ and $E\left(z ; f^{\prime}\right) \subset E\left(z ; f^{\prime \prime}\right)$, then $f \equiv f^{\prime}$.

The purpose of the paper is to further extend Theorem $\mathrm{C}$ and prove the following theorem.

THEOREM 1.1. Let $f$ be a non-constant entire function and $a(z)=$ $\alpha z+\beta$, where $\alpha(\neq 0)$ and $\beta$ are constants. If $E(a ; f) \subset E\left(a ; f^{\prime}\right)$ and $E\left(a ; f^{\prime}\right) \subset E\left(a ; f^{\prime \prime}\right)$, then either $f=A \exp \{z\}$ or

$$
f=\alpha z+\beta+(\alpha z+\beta-2 \alpha) \exp \left\{\frac{\alpha z+\beta-2 \alpha}{\alpha}\right\},
$$

where $A$ is a non-zero constant.

Corollary 1.1. If in Theorem 1.1 we assume $E(a ; f)=E\left(a ; f^{\prime}\right)$, then $f=A \exp \{z\}$, where $A$ is a non-zero constant.

Let $f, g, a$ and $b$ be meromorphic functions in $\mathbb{C}$. We denote by $N(r, a ; f \mid$ $g \neq b$ ) the integrated counting functions of those zeros of $f-a$ (counted with multiplicities) which are not the zeros of $g-b$.

For the standard definitions and notations of value distribution theory we refer the reader to [2].

2. Lemma. In this section we prove a lemma which is required to prove the theorem.

LEMMA 2.1. Let $f$ be a transcendental entire function and $a=a(z)$ $(\not \equiv 0, \infty)$ be a non-constant small function of $f$ such that $E(a ; f) \subset E\left(a ; f^{\prime}\right)$ and $E\left(a ; f^{\prime}\right) \subset E\left(a ; f^{\prime \prime}\right)$. Then $f=A \exp \{z\}$ if and only if $m(r, 1 /(f-a))=$ $S(r, f)$, where $A$ is a non-zero constant.

Proof. Since the "only if" part easily follows from Nevanlinna's three small functions theorem, we prove the "if" part.

We suppose that

$$
m\left(r, \frac{1}{f-a}\right)=S(r, f) .
$$

Let

$$
\phi=\frac{f^{\prime \prime}-f^{\prime}}{f-a} \quad \text { and } \quad \psi=\frac{\left(a-a^{\prime}\right) f^{\prime \prime}-a\left(f^{\prime}-a^{\prime}\right)}{f-a} .
$$

Also set $E=\left\{z:\left(a(z)-a^{\prime}(z)\right)\left(a(z)-a^{\prime \prime}(z)\right)=0\right\}$. Since a zero of $f-a$ which does not belong to $E$ is a simple zero, it is not a pole of $\phi$ and $\psi$. Hence $N(r, \phi)=S(r, f)$ and $N(r, \psi)=S(r, f)$. Also for any positive integer 
$p$ we get, by (2.1),

$$
\begin{aligned}
m\left(r, \frac{f^{(p)}}{f-a}\right) & =m\left(r, \frac{f^{(p)}-a^{(p)}}{f-a}+\frac{a^{(p)}}{f-a}\right) \\
& \leq m\left(r, \frac{f^{(p)}-a^{(p)}}{f-a}\right)+m\left(r, \frac{1}{f-a}\right)+m\left(r, a^{(p)}\right)+O(1) \\
& =S(r, f) .
\end{aligned}
$$

Hence $m(r, \phi)=S(r, f)$ and $m(r, \psi)=S(r, f)$. Therefore $T(r, \phi)=S(r, f)$ and $T(r, \psi)=S(r, f)$. We now consider the following two cases.

CAse I. Let $\phi \equiv 0$. Then $f^{\prime} \equiv f^{\prime \prime}$ and so $f=A \exp \{z\}+B$, where $A(\neq 0)$ and $B$ are constants. Hence $f=f^{\prime}+B$. By (2.1) there exists $z_{1}$ such that $a\left(z_{1}\right) \neq \infty$ and $a\left(z_{1}\right)=a\left(z_{1}\right)+B$ and so $B=0$. Therefore $f=A \exp \{z\}$.

CASE II. Let $\phi \not \equiv 0$. Let $z_{0}$ be a zero of $f-a$ and $z_{0} \notin E$. Then in some neighbourhood of $z_{0}$ we get

$$
\begin{aligned}
f & =a\left(z_{0}\right)+a_{1}\left(z-z_{0}\right)+a_{2}\left(z-z_{0}\right)^{2}+a_{3}\left(z-z_{0}\right)^{3}+O\left(\left(z-z_{0}\right)^{4}\right), \\
f^{\prime} & =a_{1}+2 a_{2}\left(z-z_{0}\right)+3 a_{3}\left(z-^{\prime} z_{0}\right)^{2}+O\left(\left(z-z_{0}\right)^{3}\right), \\
f^{\prime \prime} & =2 a_{2}+6 a_{3}\left(z-z_{0}\right)+O\left(\left(z-z_{0}\right)^{2}\right)
\end{aligned}
$$

where $a_{1}=2 a_{2}=a\left(z_{0}\right)$ and $6 a_{3}=f^{(3)}\left(z_{0}\right)$.

So in some neighbourhood of $z_{0}$ we obtain

$$
\begin{aligned}
\phi & =\frac{\left(6 a_{3}-2 a_{2}\right)\left(z-z_{0}\right)+O\left(\left(z-z_{0}\right)^{2}\right)}{a\left(z_{0}\right)-a(z)+a_{1}\left(z-z_{0}+O\left(\left(z-z_{0}\right)^{2}\right)\right.} \\
& =\frac{\left(6 a_{3}-2 a_{2}\right)\left(z-z_{0}\right)+O\left(\left(z-z_{0}\right)^{2}\right)}{\left(a_{1}-a^{\prime}\left(z_{0}\right)+o(1)\right)\left(z-z_{0}\right)+O\left(\left(z-z_{0}\right)^{2}\right)} \\
& =\frac{6 a_{3}-2 a_{2}+O\left(z-z_{0}\right)}{a_{1}-a^{\prime}\left(z_{0}\right)+o(1)+O\left(z-z_{0}\right)} .
\end{aligned}
$$

Hence

$$
\phi\left(z_{0}\right)=\frac{f^{(3)}\left(z_{0}\right)-a\left(z_{0}\right)}{a\left(z_{0}\right)-a^{\prime}\left(z_{0}\right)} .
$$

Also in some neighbourhood of $z_{0}$ we get

$$
\psi=\frac{a^{\prime}(z) a^{\prime}\left(z_{0}\right)+f^{(3)}\left(z_{0}\right)\left(a(z)-a^{\prime}(z)\right)-a(z) a\left(z_{0}\right)+O\left(z-z_{0}\right)}{a\left(z_{0}\right)-a^{\prime}\left(z_{0}\right)+o(1)+O\left(z-z_{0}\right)} .
$$

Hence

$$
\psi\left(z_{0}\right)=f^{(3)}\left(z_{0}\right)-a\left(z_{0}\right)-a^{\prime}\left(z_{0}\right) .
$$

From (2.2) and (2.3) we get

$$
\left\{a\left(z_{0}\right)-a^{\prime}\left(z_{0}\right)\right\} \phi\left(z_{0}\right)-\psi\left(z_{0}\right)-a^{\prime}\left(z_{0}\right)=0 .
$$


If $\left(a-a^{\prime}\right) \phi-\psi-a^{\prime} \not \equiv 0$, then from (2.4) we get

$$
N\left(r, \frac{1}{f-a}\right) \leq N\left(r, 0 ;\left(a-a^{\prime}\right) \phi-\psi-a^{\prime}\right)+S(r, f)=S(r, f),
$$

which together with (2.1) implies $T(r, f)=S(r, f)$, a contradiction. Therefore

$$
\left(a-a^{\prime}\right) \phi-\psi \equiv a^{\prime}
$$

First we suppose that $\psi \equiv 0$. Then from (2.5) and the definitions of $\phi$ and $\psi$ we get

$$
\left(a-a^{\prime}\right) \frac{f^{\prime \prime}-f^{\prime}}{f-a} \equiv a^{\prime}
$$

and

$$
\left(a-a^{\prime}\right) f^{\prime \prime} \equiv a\left(f^{\prime}-a^{\prime}\right) .
$$

From (2.6) and (2.7) we obtain $f \equiv f^{\prime}$ and so $\phi \equiv 0$, which is a contradiction.

Next we suppose that $\psi \not \equiv 0$. Then from (2.5) and the definitions of $\phi$ and $\psi$ we get

$$
\left(a-a^{\prime}\right) \frac{f^{\prime \prime}-f^{\prime}}{f-a}-\frac{\left(a-a^{\prime}\right) f^{\prime \prime}-a\left(f^{\prime}-a^{\prime}\right)}{f-a} \equiv a^{\prime} .
$$

This implies $f \equiv f^{\prime}$ and so $\phi \equiv 0$, which is a contradiction. This proves the lemma.

3. Proofs of the theorem and corollary. In this section we prove the main result of the paper.

Proof of Theorem 1.1. First we suppose that $f$ is a polynomial and consider the following cases.

CAse I. Let $f=A z+B$, where $A(\neq 0)$ and $B$ are constants. If $z_{0}$ is a zero of $f-a$, then by the hypotheses $z_{0}$ is also a zero of $f^{\prime}-a$ and $f^{\prime \prime}-a$. Hence $A=a\left(z_{0}\right)=0$, a contradiction.

CASE II. Let $f=A z^{2}+B z+C$, where $A(\neq 0), B$ and $C$ are constants. If $f(z)-a(z)=0$ has two distinct roots, then $E(a ; f) \subset E\left(a ; f^{\prime}\right)$ implies that $f^{\prime}(z) \equiv a(z)$. Again since $E\left(a ; f^{\prime}\right) \subset E\left(a ; f^{\prime \prime}\right)$, we arrive at a contradiction. So $f(z)-a(z)=0$ has only one double root. Also $E(a ; f) \subset E\left(a ; f^{\prime}\right)$ implies that if this root is $z_{0}$ then $a\left(z_{0}\right)=a^{\prime}\left(z_{0}\right)$ and so $z_{0}=(\alpha-\beta) / \alpha$. Since $f^{\prime \prime}\left(z_{0}\right)=a\left(z_{0}\right)$, we get $\alpha=2 A$. Also $f^{\prime}\left(z_{0}\right)=a\left(z_{0}\right)$ implies $B=\beta$ and $f\left(z_{0}\right)=a\left(z_{0}\right)$ implies $C=\left(\alpha^{2}+\beta^{2}\right) / 2 \alpha$. Therefore

$$
f(z)=\frac{\alpha}{2} z^{2}+\beta z+\frac{\alpha^{2}+\beta^{2}}{2 \alpha}
$$

and so $f^{\prime}(z) \equiv a(z)$. Since $E\left(a ; f^{\prime}\right) \subset E\left(a ; f^{\prime \prime}\right)$, we arrive at a contradiction. 
CASE III. Let $f$ be a polynomial of degree $d(\geq 3)$. If $z_{1}, \ldots, z_{n}$ are the roots of the equation $f(z)-a(z)=0$, we can write

$$
f(z)=a(z)+A\left(z-z_{1}\right)^{p_{1}} \cdots\left(z-z_{n}\right)^{p_{n}},
$$

where $p_{1}+\cdots+p_{n}=d$ and $A(\neq 0)$ is a constant.

Also by the hypotheses

$$
f^{\prime}(z)=a(z)+B\left(z-z_{1}\right)^{q_{1}} \cdots\left(z-z_{n}\right)^{q_{n}} Q(z)
$$

and

$$
f^{\prime \prime}(z)=a(z)+C\left(z-z_{1}\right)^{r_{1}} \cdots\left(z-z_{n}\right)^{r_{n}} Q(z) R(z),
$$

where $Q, R$ are polynomials such that $q_{1}+\cdots+q_{n}+\operatorname{deg} Q=d-1$, $r_{1}+\cdots+r_{n}+\operatorname{deg} Q+\operatorname{deg} R=d-2$ and $B(\neq 0), C$ are constants.

First we suppose that $C=0$. Then $f^{\prime \prime}(z) \equiv a(z)$ and so

$$
f(z)=\frac{\alpha^{2}}{6} z^{3}+\frac{\beta}{2} z^{2}+\gamma z+\delta \quad \text { and } \quad f^{\prime}(z)=\frac{\alpha}{2} z^{2}+\beta z+\gamma,
$$

where $\gamma, \delta$ are constants. Since $E(a ; f) \subset E\left(a ; f^{\prime}\right)$, we see that $f(z)-a(z)=0$ must have one multiple root, say $z_{0}$. If its multiplicity is three, then by the hypotheses we have $a\left(z_{0}\right)=a^{\prime}\left(z_{0}\right)=a^{\prime \prime}\left(z_{0}\right)$, which is impossible because $\alpha \neq 0$. So $f(z)-a(z)=0$ has one double root and it is a root of $a(z)-$ $a^{\prime}(z)=0$. Hence $z=(\alpha-\beta) / \alpha$ is a double root of $f(z)-a(z)=0$. Also it is a root of $f^{\prime}(z)-a(z)=0$ and so $\gamma=\left(\alpha^{2}+\beta^{2}\right) / 2 \alpha$. Hence

$$
f^{\prime}(z)-a(z)=\frac{\alpha}{2}\left(z-\frac{\alpha-\beta}{\alpha}\right)^{2} .
$$

Since $E(a ; f) \subset E\left(a ; f^{\prime}\right)$ and $f(z)-a(z)=0$ has two distinct roots, we arrive at a contradiction. Therefore $C \neq 0$.

Since $E(a ; f) \subset E\left(a ; f^{\prime}\right)$, we see that the roots of $f(z)-a(z)=0$ cannot all be simple. By the hypotheses we see that a multiple root of $f(z)-a(z)=0$ must be a root of $a(z)-a^{\prime}(z)=0$ and so it is $(\alpha-\beta) / \alpha$. If its multiplicity is greater than two, then it is a root of $a(z)-a^{\prime \prime}(z)=0$ and so $\alpha=0$, which is impossible. So $z=(\alpha-\beta) / \alpha$ is a double root of $f(z)-a(z)=0$. Without loss of generality we put $z_{1}=(\alpha-\beta) / \alpha$ and $p_{1}=2$. Then $z_{2}, \ldots, z_{n}$ are all simple roots of $f(z)-a(z)=0$. Therefore $d=n+1$ and so $q_{1}=\cdots=q_{n}=1$ and $\operatorname{deg} Q=0$. Since $E\left(a ; f^{\prime}\right) \subset E\left(a ; f^{\prime \prime}\right)$, we get $r_{j} \geq 1$ for $j=1, \ldots, n$. Hence $n+\operatorname{deg} R \leq r_{1}+\cdots+r_{n}+\operatorname{deg} R=n-1$, which is a contradiction.

Therefore $f$ is a transcendental entire function. Let

$$
\psi=\frac{\left(a-a^{\prime}\right) f^{\prime \prime}-a\left(f^{\prime}-a^{\prime}\right)}{f-a} .
$$

If $\psi \equiv 0$, then

$$
\frac{f^{\prime \prime}}{f^{\prime}-\alpha} \equiv 1+\frac{\alpha}{\alpha z+\beta-\alpha}
$$


This gives on integration $f^{\prime}=\alpha+A(\alpha z+\beta-\alpha) \exp \{z\}$ and $f=\alpha z+$ $A(\alpha z+\beta-2 \alpha) \exp \{z\}+B$, where $A(\neq 0)$ and $B$ are constants. Also $f^{\prime \prime}=A(\alpha z+\beta) \exp \{z\}$. Since $E(a ; f) \subset E\left(a ; f^{\prime}\right)$ and $E\left(a ; f^{\prime}\right) \subset E\left(a ; f^{\prime \prime}\right)$, we see that $f(z)-a(z)=0$ has the unique solution $z_{0}=(2 \alpha-B) / \alpha$. Also $f(z)-(\alpha z+B)=0$ has only one solution $z_{1}=(2 \alpha-\beta) / \alpha$. Hence by Nevanlinna's three small functions theorem we get $B=\beta$. So

$$
f=\alpha z+\beta+A(\alpha z+\beta-2 \alpha) \exp \{z\} .
$$

Also since $E(a ; f) \subset E\left(a ; f^{\prime}\right)$, it follows that $\alpha+A\left(\alpha z_{0}+\beta-\alpha\right) \exp \left\{z_{0}\right\}=$ $\alpha z_{0}+\beta$ and so $A=\exp \{(\beta-2 \alpha) / \alpha\}$. Therefore

$$
f=\alpha z+\beta+(\alpha z+\beta-2 \alpha) \exp \left\{\frac{\alpha z+\beta-2 \alpha}{\alpha}\right\} .
$$

Now we suppose that $\psi \not \equiv 0$. Then

$$
f-a \equiv \frac{1}{\psi}\left[\left(a-a^{\prime}\right) f^{\prime \prime}-a\left(f^{\prime}-a^{\prime}\right)\right]
$$

and so

$$
\begin{aligned}
{\left[1+a\left(\frac{1}{\psi}\right)^{\prime}+\frac{a^{\prime}}{\psi}\right] } & \left(f^{\prime}-a\right) \equiv\left(a^{\prime}-a\right)\left[1+\left(\frac{1}{\psi}\right)^{\prime}\left(a-a^{\prime}\right)+\frac{2 a^{\prime}}{\psi}\right] \\
& +\left(a^{\prime}-a\right)\left[\frac{1}{\psi}-\left(\frac{1}{\psi}\right)^{\prime}\right]\left(f^{\prime \prime}-a^{\prime}\right)-\left(a^{\prime}-a\right) \frac{f^{\prime \prime \prime}}{\psi} .
\end{aligned}
$$

Let

$$
\Delta=1+\left(\frac{1}{\psi}\right)^{\prime}\left(a-a^{\prime}\right)+\frac{2 a^{\prime}}{\psi} \equiv 0 .
$$

Then

$$
\psi^{2}+2 \alpha \psi \equiv \psi^{\prime}(\alpha z+\beta-\alpha) .
$$

If $\psi$ is transcendental, then from (3.2) we get

$$
T(r, \psi)=m(r, \psi)+N(r, \psi) \leq m\left(r, \psi^{\prime} / \psi\right)+O(\log r)=S(r, \psi),
$$

a contradiction.

Hence $\psi$ is a rational function. If $\psi$ has a pole, then by the hypotheses we see that $z=(\alpha-\beta) / \alpha$ is the only pole of $\psi$. If $p$ is its multiplicity, then from (3.2) we get $2 p=p$. So $\psi$ has no pole at all. If $n$ is the degree of $\psi$, then from (3.2) we get $2 n=n$ and so $n=0$. Hence $\psi$ is a constant and from (3.2) we get $\psi=-2 \alpha$. Therefore

$$
(\alpha z+\beta-\alpha) f^{\prime \prime}-(\alpha z+\beta)\left(f^{\prime}-\alpha\right)+2 \alpha(f-\alpha z-\beta) \equiv 0 .
$$

Differentiating twice we get

$$
\frac{f^{(4)}}{f^{(3)}}=1-\frac{\alpha}{\alpha z+\beta-\alpha} .
$$


On integration we obtain

$$
f^{(3)}=\frac{A}{\alpha z+\beta-\alpha} \exp \{z\},
$$

where $A(\neq 0)$ is a constant. This is impossible because $f$ is entire. Therefore $\Delta \not \equiv 0$ and so from (3.1) we get

$$
\frac{1}{f^{\prime}-a} \equiv \frac{1+a\left(\frac{1}{\psi}\right)^{\prime}+\frac{a^{\prime}}{\psi}}{\left(a^{\prime}-a\right) \Delta}-\frac{\frac{1}{\psi}-\left(\frac{1}{\psi}\right)^{\prime}}{\Delta} \cdot \frac{f^{\prime \prime}-a^{\prime}}{f^{\prime}-a}+\frac{1}{\psi \Delta} \cdot \frac{f^{\prime \prime \prime}}{f^{\prime}-a} .
$$

Since $T(r, \psi)=S(r, f)$ and $f$ is transcendental, we get

$$
m\left(r, \frac{1}{f^{\prime}-a}\right)=S(r, f) \text {. }
$$

By the hypotheses we see that $z=(\alpha-\beta) / \alpha$ is the only possible multiple (actually double) zero of $f^{\prime}-a$. So $N\left(r, a ; f^{\prime} \mid f \neq a\right) \leq N(r, 0 ; \psi)+$ $O(\log r)=S(r, f)$. Therefore

$$
\begin{aligned}
N\left(r, a ; f^{\prime}\right) & =N(r, a ; f)+N\left(r, a ; f^{\prime} \mid f \neq a\right)+O(\log r) \\
& =N(r, a ; f)+S(r, f) .
\end{aligned}
$$

Again since $f$ is entire and

$$
f=a+\frac{f^{\prime}-a^{\prime}}{\psi}\left[\left(a-a^{\prime}\right) \frac{f^{\prime \prime}}{f^{\prime}-a^{\prime}}-a\right],
$$

we get

$$
\begin{aligned}
T(r, f) & =m(r, f) \leq m\left(r, f^{\prime}-a^{\prime}\right)+S(r, f) \\
& \leq m\left(r, f^{\prime}\right)+S(r, f)=T\left(r, f^{\prime}\right)+S(r, f) .
\end{aligned}
$$

Also

$$
T\left(r, f^{\prime}\right)=m\left(r, f^{\prime}\right) \leq m(r, f)+m\left(r, f^{\prime} / f\right)=T(r, f)+S(r, f) .
$$

Therefore

$$
T(r, f)=T\left(r, f^{\prime}\right)+S(r, f) .
$$

From (3.3)-(3.5) we get

$$
\begin{aligned}
m\left(r, \frac{1}{f-a}\right) & =T(r, f)-N\left(r, \frac{1}{f-a}\right)+S(r, f) \\
& =T\left(r, f^{\prime}\right)-N\left(r, \frac{1}{f-a}\right)+S(r, f) \\
& =N\left(r, \frac{1}{f^{\prime}-a}\right)-N\left(r, \frac{1}{f-a}\right)+S(r, f) \\
& =S(r, f) .
\end{aligned}
$$

Therefore by Lemma 2.1 we get $f=A \exp \{z\}$. This proves the theorem. 
Proof of Corollary 1.1. If

$$
f=(\alpha z+\beta)+(\alpha z+\beta-2 \alpha) \exp \left\{\frac{\alpha z+\beta-2 \alpha}{\alpha}\right\},
$$

then we see that $E(a ; f)$ contains only one element but $E\left(a ; f^{\prime}\right)$ contains infinitely many elements. This contradicts the hypothesis $E(a ; f)=E\left(a ; f^{\prime}\right)$. Therefore by Theorem 1.1 we get $f=A \exp \{z\}$. This proves the corollary.

\section{References}

[1] J. Chang and M. Fang, Uniqueness of entire functions and fixed points, Kodai Math. J. 25 (2002), 309-320.

[2] W. K. Hayman, Meromorphic Functions, Clarendon Press, Oxford, 1964.

[3] G. Jang, E. Mues und L. Volkman, Meromorphe Funktionen, die mit ihrer ersten und zweiten Ableitung einen endlichen Wert teilen, Complex Variables Theory Appl. 6 (1986), 51-71.

[4] L. A. Rubel and C. C. Yang, Values shared by an entire function and its derivative, in: Complex Analysis (Kentucky, 1976), Lecture Notes in Math. 599, Springer, 1977, 101-103.

Department of Mathematics

University of Kalyani

West Bengal 741235, India

E-mail: indr9431@dataone.in
Department of Mathematics

G.M.S.M. Mahavidyalaya Bireswarpur, South 24 Parganas, India

E-mail: g80g@rediffmail.com

Received 4.10.2008

and in final form 14.1.2009 